\title{
EXPERIMENTAL STUDY OF 3D SELF-ASSEMBLED PHOTONIC CRYSTALS AND COLLOIDAL CORE-SHELL SEMICONDUCTOR QUANTUM DOTS
}

\author{
Pham Thu Nga* \\ Institute of Materials Science, Vietnamese Academy of Science and Technology \\ Faculty of Engineering Physics and Nano -Technology, College of Technology \\ Vu Duc Chinh, Nguyen Xuan Nghia, Nguyen Viet Huy \\ Institute of Materials Science, VAST, Vietnam
}

Dao Nguyen Thuan

Faculty of Engineering Physics and Nano -Technology, College of Technology, Vietnam

Pham Thai Cuong, Chu Viet Ha, Do Thuy Chi

Thai Nguyen Pedagogical University, Thai Nguyen, Vietnam

Nguyen Nhu Dat

Institute of Physics and Electronics, VAST, Vietnam

Le Lan Anh

Institute of Chemistry, VAST, Vietnam

C. Barthou, P. Benalloul, M. Romanelli, A. Maître

Institut des Nano Sciences de Paris (INSP), UMR - CNRS 7588, France

Received 27 November 2006

\begin{abstract}
In this contribution we present an experimental study of 3D opal photonic crystals. The samples are opals constituted by colloidal silica spheres, realized with self-assembly technique. The sphere diameter is selected in order to obtain coupling of the photonic band gap with the
\end{abstract}

*Corresponding author e-mail: phtnga@ims.vast.ac.vn 
emission from $\mathrm{CdSe} / \mathrm{ZnS}$ colloidal quantum dots. The quantum dots infiltrated in the opals is expected to be enhanced or suppressed depending on the detection angle from the photonic crystal. The structural and optical characterization of the $\mathrm{SiO}_{2}$ opal photonic crystals are performed by field-emission scanning electron microscopy and reflectivity spectroscopy. Measurements performed on samples permits to put into evidence the influence of the different preparation methods on the optical properties. Study of self-activated luminescence of the pure opals is also presented. It is shown that the luminescence of the sample with QDs have original QD emission and not due to the photonic crystal structure. The optical properties of colloidal core-shell semiconductor quantum dots of $\mathrm{CdSe} / \mathrm{ZnS}$ which are prepared in our lab will be mention.

Keywords: Photonic crystals, $\mathrm{SiO}_{2}$ opals, $\mathrm{CdSe} / \mathrm{ZnS}$ quantum dots, optical properties

\section{INTRODUCTION}

Three-dimensional photonic crystals (PCs) and photonic-band gap (PBG) materials are a unique class of materials with a periodically modulated dielectric constant. These ordered dielectric structures with sizes comparable to the wavelength of electromagnetic (EM) waves have attracted considerable interest for possible applications because the propagation of EM waves can be manipulated by defining allowed and forbidden energy gaps in the photon dispersion spectrum [1]. The absence of propagation of EM modes inside the structures gives rise to distinct optical phenomena. One of the representative bottom-up approaches used to prepare 3D photonic crystal structures is the spontaneous self-organization of spherical colloidal particles. This approach has been proposed as a possible route for the creation of PBG structures $[2,3]$. Such artificial opal structures have been widely studied because of the ease and low-cost process for growing a 3D periodic structure. Recently, PCs have attracted much attention from both fundamental and practical points of view, because novel physical concepts such as photonic band gap have been theoretically predicted and various applications of photonic crystals have been proposed.

Modern technology of colloidal nanocrystal preparation allows the fabrication of spherical semiconductor quantum dots (QD) with narrow size distribution $[4,5]$. Colloidal quantum dots having wide excitation spectra, narrow emission spectra, high quantum yields for luminescence, and great photo stability are ideal laser material $[6,7]$ single-photon source $[8]$ and luminescent labels for chemical and potential biological applications [9]. Photonic crystals based on artificial colloidal silica crystals (opals) exhibit pronounced stop bands for electromagnetic wave propagation and the corresponding modification of the photon density of states in the visible range. The opal is a cubic-close-packed lattice of $\mathrm{SiO}_{2}$ spheres. Doping of these structures with semiconductor quantum dots provides a possibility to examine the predictions of the inhibited spontaneous emission in photonic band-gap materials.

The aim of this work is the synthesis of silica colloid particles, and fabrication of the opal photonic crystal, the infiltration of CdSe/ZnS QDs into the opal photonic crystal and characterization of the resulting this composite structure.

First, we report on the synthesis of $\mathrm{SiO}_{2}$ colloid particles with sub micrometer sizes, which were used as the building blocks for the fabrication of opal photonic crystals. Using convective selfassembly, colloid particles will be assembled under optimized conditions, such as temperature and particle concentration in the solution. Opal photonic crystals with sharp-edged and convenient stop bands will be selected for the infiltration of CdSe/ZnS QD. In the second part, we report on optical characterization of $\mathrm{CdSe} / \mathrm{ZnS}$ QDs in this structure by photoluminescence (PL). We explored ways to infiltrate $\mathrm{CdSe} / \mathrm{ZnS}$ QDs into the opaline photonic crystal. We considered the use of capillary forces to infiltrate CdSe/ZnS QDs into interstitial spaces. The infiltration of $\mathrm{CdSe} / \mathrm{ZnS}$ QDs in solution into photonic crystals is carried out by immersion. The 
measurement of photoluminescence of the infiltrated CdSe/ZnS QDs opal photonic crystals is performed.

\section{EXPERIMENTS SECTION}

\subsection{Synthesis of silica colloid particles and fabrication of $\mathrm{SiO}_{2}$ opal photonic crystals}

Artificial silica colloidal crystals possess a three-dimensional periodicity on the submicron scale resulting in iridescent colors related to the interference of light. The spontaneous crystallization of colloids (hard particles in suspension) provides a much simpler, faster and cheaper alternative to nanolithography $[2,10]$. This approach involves the 'natural' self-assembly of silica micro spheres from colloidal suspension into a solid, three-dimensionally periodic structure, which can then act as a template for construction of the photonic crystal. The template contains voids that can be infiltrated by a material of high refractive index, and removal of the original template by wet etching (by dissolving with acid for example) leads to the desired photonic crystal structure [11].

The synthesis of opals includes the fabrication of monodisperse silica colloidal micro spheres. The silica spheres were prepared by hydrolysis and condensation of tetraethyl orthosilicate (TEOS) in a mixture of water, ammonia, and ethanol with proportion of 0.376 gr. TEOS; 0.805 gr. water, 0.693 gr. ammonia and 7.891 gr. ethanol. We applied used the method of Stöber to obtain the pre-determinant micro sphere size [12]. TEOS was added to the ethanol solution of ammonia (25\% $\mathrm{NH}_{3}$ solution) and water in one step and the mixture was stirred for $24 \mathrm{~h}$. The silica suspensions were centrifuged at $3000 \mathrm{rpm}$ for $30 \mathrm{~min}$ and washed with ethanol. The centrifuging/washing procedure was repeated 6 times. The particles were dried at $120^{\circ} \mathrm{C}$ overnight. By varying the molar concentrations of TEOS, ammonia, and water, we can receive different particle size. Under certain conditions $\mathrm{SiO}_{2}$ colloids may spontaneously form ordered lattices. This phenomenon is called self-assembly. In this work, we used the vertical deposition method on the substrate ( $\mathrm{Si}$ wafer or glass slide) for thin film opals.

The vertical deposition is used and has taken over from other methods due to its multiple advantages as good control over sample thickness, samples with sizes of several square centimeters can be fabricated in one or two days, samples are attached to a substrate (usually a glass or silicon slide) and its manipulation becomes easier. Since opals are much thinner, the number of defects is much lower. The infiltration of air voids with guest materials as quantum dots is easier in thin film opals since material must penetrate just a few layers. In the preparation method, Si wafer substrates $(3 \mathrm{~cm} \times 2 \mathrm{~cm})$ were used. They are carefully cleaned in a solution of $\mathrm{HF}(1 \%)$ and afterwards another of $\mathrm{H}_{2} \mathrm{SO}_{4} / \mathrm{H}_{2} \mathrm{O}_{2}$ (2:1 vol.) before use. The glass slides are cleaned in acetone. It must be noticed that conditions for glass slides would be significantly different. Finally the substrates are rinsed in doubly distilled water, acetone and ethanol. It is important note to set the substrates completely vertical (tilted 30 to 40 degrees respect to the vertical direction) or too thin opals (1 - 3 layers) are obtained (Fig. 2). Figure 3 shows an optical microscope image taken with a $4 \mathrm{X}$ objective from one of the samples fabricated with this method. The ordered structure is well seen in the reflected light as regions of the same green color from the sample. The number of layers usually varies from several to 20 layers. In some occasions the number of layers can be counted easily between different layers from SEM picture.

In the experimental setup, a clean substrate is placed into a vial containing a silica alcosol. The alcosol is a suspension containing a volume fraction ranging from $0.1 \%$ to $5 \%$ of silica spheres in ethanol. The resulting sample thickness depends on the volume fraction. The setup system was used with a gradient temperature from $80^{\circ} \mathrm{C}$ to $65^{\circ} \mathrm{C}$ as reported in $[2,1]$. In this case capillary forces cause the spheres crystallization in the meniscus formed by the alcosol and the substrate. To avoid a too fast aggregation rate, high initial volume fractions and evaporation rates must be avoided. The (111) planes are parallel to the substrate. By adding a temperature 
gradient along the suspension, on the one hand, the temperature at the surface of the suspension was low enough to obtain a valid evaporation rate. On the other hand, the temperature gradient causes convection forces and a continuous flow of spheres is directed towards the surface. Using this technique, Vlasov et al. have succeeded in ordering silica spheres $(0.86 \mathrm{~mm}$ in diameter) onto a silicon wafer [2]. The resulting structure has an order of magnitude fewer point defects and the individual silica crystals are two orders of magnitude larger than previously possible. Once the opal crystal is in place, the voids in the structure can be infiltrated with a CdSe/ZnS quantum dots.

\subsection{The structural characterization}

All the samples are measured by using field-emission scanning electron microscopy (FE-SEM, S-4800 Hitachi, Japan) to characterization of the ordered structural. It was used also to identify the thickness and the crystal quality of the opal structures. For measurement of the optical reflection spectra, we use the system with the light from a single-mode optical fiber from a white light source was focused on the sample using an objective lens. The light reflected from the sample was focused on a multimode fiber coupled to a spectrometer. These two optical fibers can be turned to an angle. The sample is illuminated with a broadband source (halogen lamp and deuterium lamp) at various incidence angles. The reflected light is collected at specular reflection and spectrally analyzed. All the reflectivity spectra are normalized to the lamp spectrum. The PL spectra are performed for the opal samples and the samples with CdSe/ZnS QDs.

\section{RESULTS AND DISCUSSION}

\subsection{Structural characterization of the opal photonic crystals}

Figure 1 ( $a, b, c, d, e, f)$ shows FE-SEM images of particle sizes, of the cleaved edge of opal crystal (Fig. 1b, c, d) and of the surface of the sample, it is the structural characterization. The size of silicon dioxide spheres ranged from $255 \mathrm{~nm}$ (Fig. 1a) to $287 \mathrm{~nm}$ (Fig. 1e) for different samples. By using our home-made silica spheres of $\sim 287 \mathrm{~nm}$ diameter, we prepared the opal photonic crystals which were dried naturally at room temperature for about one month (Fig. 1e). These FE-SEM images show the diameter and homogenous of the sphere size from different sample. We can preparer also the sphere with the size up to 460 and $870 \mathrm{~nm}$.

By the SEM images, one can say that we have prepared $\mathrm{SiO}_{2}$ opals of high quality, achieved by a procedure of stricter selection of $\mathrm{SiO}_{2}$ spheres. We confirm that good monodispersity of the spheres is one of the major factors for obtaining opals with fine optical properties and large size. A recipe will be developed to fabricate thin film silica opals on silicon substrates made of spheres with a diameter close to $850 \mathrm{~nm}$ to prove how useful this method could be. The thickness of samples can achieve to about $\sim 13 \mu \mathrm{m}$ (Fig. 1f). Samples are attached to a glass substrate or silicon slide.

\subsection{Optical properties of the opal photonic crystals}

As presented above, Scanning Electron Microscopy (SEM) allowed obtaining some information about the structural properties of the samples. Unfortunately this technique is destructive and the amount of information extracted does not provide a rich enough description of the opal properties. The lattice parameter and the refractive index (RI) are two of these characteristics for which SEM does not provide an accurate information. The techniques based on optical characterization will be introduced to complete the photonic crystal description. As a structure where the refractive index is modulated, the optical response of an opal varies depending on the direction studied. Even though the RI contrast is rather low for silica opals (1.425:1.000), the 164 
anisotropy effects, especially at high energies, make the data interpretation a non trivial issue. Specular reflectance spectra are presented in this section to show that these opals have single crystalline domains. We think that the quality of these samples is due to a meniscus-induced shear that aligns the close-packed layers into a f.c.c. crystal during deposition.

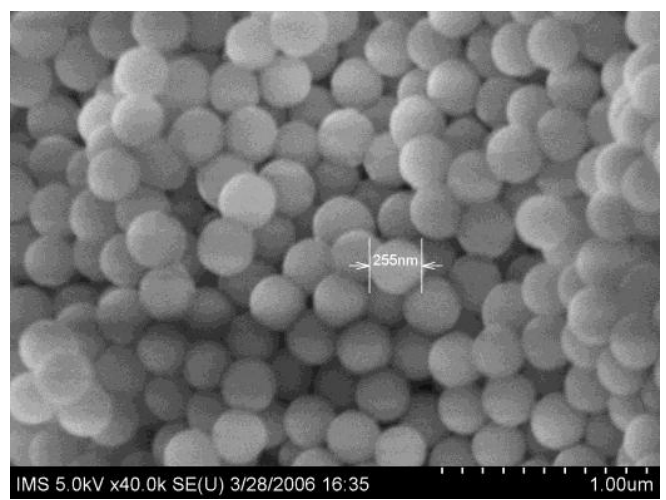

(a)

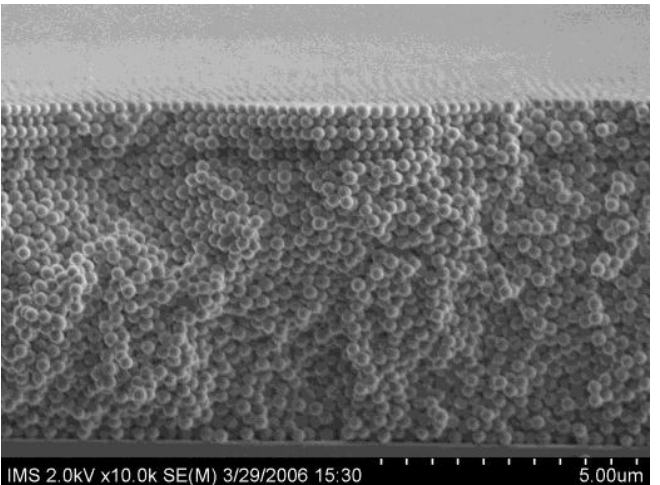

(c)

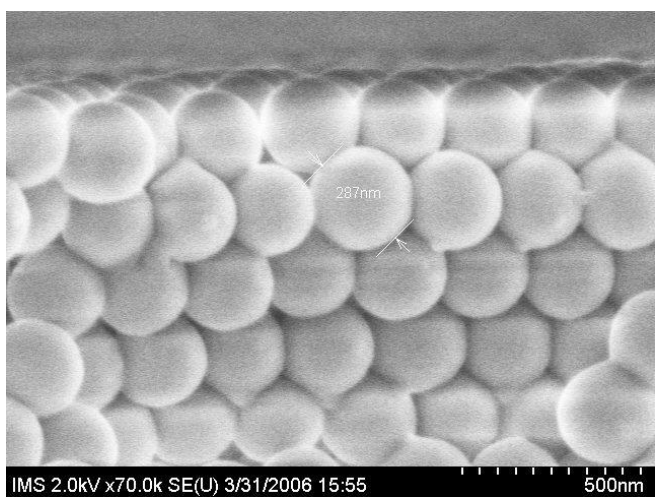

(e)

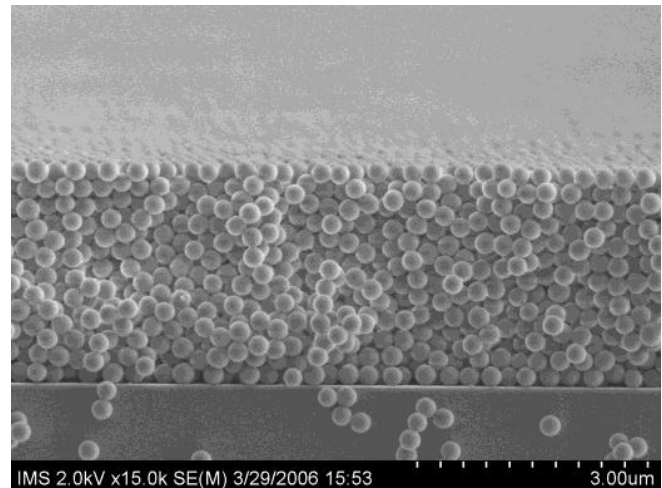

(b)

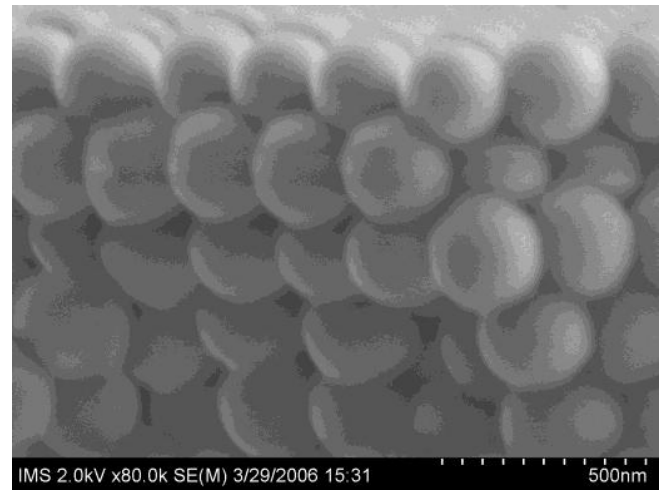

(d)

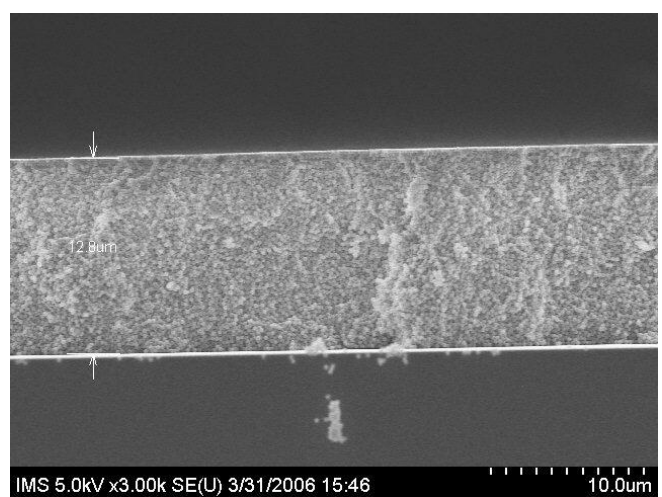

(f)

Fig. 1: SEM images of thin film opal made from $287 \mathrm{~nm}$ silica spheres. a) the diameter of the $\mathrm{SiO}_{2}$ spheres; b) top (111) surface for the opal thin film; c) for the thick film, d) and e) high-magnification image of the cleaved edge of opal crystal about 14 layers thick; $f$ ) low-magnification image of the cleaved edge of opal crystal to show the thickness is $12.8 \mu \mathrm{m}$ 


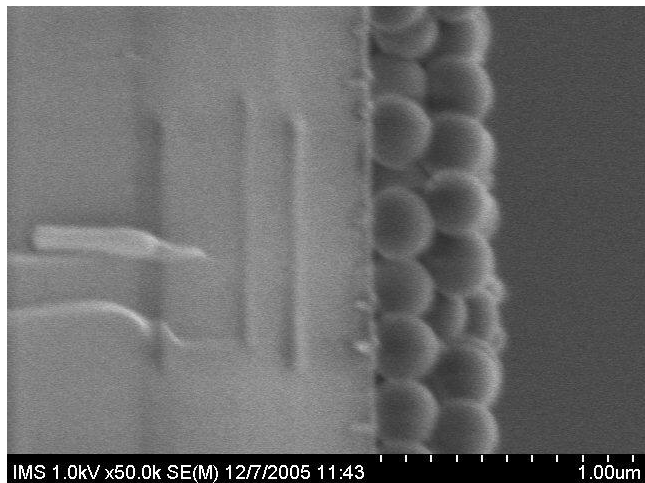

Fig. 2: SEM high-magnification image of the cleaved edge of opal crystal with 2 layers

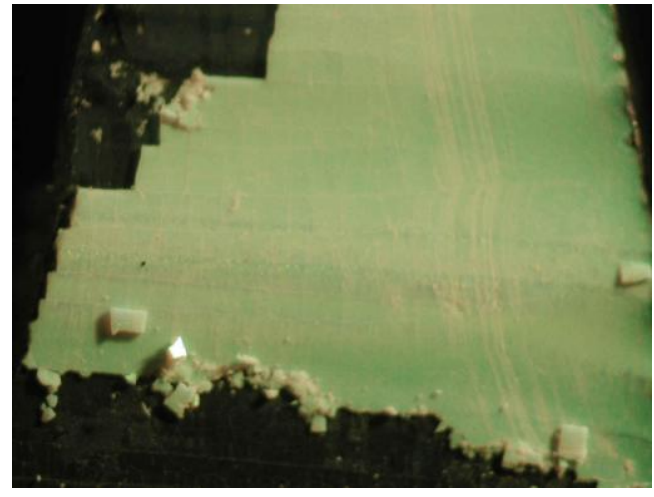

Fig. 3: Optical microscope image taken with a 4 X objective of the opal sample

We explored the existence of the photonic bandgap in such crystals using optical spectroscopy. We first examined the photonic properties of the unfilled opal. An artificial opal made of silica spheres does not present a complete photonic band gap (cPBG) [13]. However, for some directions and energies there are regions not covered by any band, these are the so called pseudo gaps. These pseudo gaps do not allow the propagation of light through the opal and cause transmission dips or reflectance peaks in the sample spectra. The studying of the experimental behavior of the pseudo gaps helps to characterize the artificial opal and gives information about the optically relevant parameters (i.e. structure, topography, lattice parameter, refractive index, etc.). The multilayer films show photonic band gap properties, which can be detected by reflectance measurements in the visible.

\subsubsection{Specular reflectance spectra}

Reflection spectra were measured at room temperature by a spectrometer equipped with two optical fibers: one for guided incident light, another one for collection of all reflected light from the opal sample. The spectra were measured at normal incidence of light onto the $\{111\}$ plane of crystals in the spectral range from 400 to $900 \mathrm{~nm}$ and at different angles with the normal incidence as $20^{\circ}, 30^{\circ}, 40^{\circ}, 50^{\circ}, 60^{\circ}, 70^{\circ}$. In the examined range the reflection spectra of all samples exhibit the Bragg diffraction peak. Figure 4 shows the reflection spectra of the opals, the spectral position of the reflection maximum at $\lambda=555 \mathrm{~nm}$ in the sample of the opal corresponds to the Bragg condition $\lambda=2 d n_{\text {eff }}$ with the effective value of refraction index $n_{\text {eff }}=$ 1.37 in agreement with the calculation from formula. In our case of the face-centered cubic lattice the plane-to-plane distance is $d=0.816 D_{\mathrm{SiO} 2}$ along the $\{111\}$ direction, $D_{\mathrm{SiO} 2}=253$ and $285 \mathrm{~nm}$ being the diameters of the $\mathrm{SiO}_{2}$ spheres in the opal samples. It should be taken into account that for the initial 'raw' opal the volumetric proportion of $\mathrm{SiO}_{2}$ is $f=0.86$. Figure 4 shows the reflectance spectra dependence of the incident angle.

Bragg's law allows us to experimentally study all the directions. It would provide a lot of useful information about the photonic crystal. Opals are usually grown in the $\{111\}$ direction and, therefore, only the optical properties around the $\Gamma L$ direction can be probed. We can apply Bragg's law and regarding spheres as point scatterers forming planes. These planes scatter light and for certain directions and wavelengths these scattered waves are added up. The equation can be obtained in the same way where it is obtained for X-rays. But in this case it must be taken into account that the photonic crystal has a refractive index different to that of air. When the light satisfies Bragg conditions, it is diffracted away from the light-propagation $\mathrm{k}$ vector 
crossing the Brillouin zone boundaries in the vicinity of the L points. For the light with a certain incident angle, the Bragg reflection wavelength $\lambda_{\max }$ can be determined using [14]:

$$
\lambda=2 d \sqrt{\left[n_{c}-n_{m} \sin ^{2}\left(\theta_{e x t}\right)\right]},
$$

where $d$ is the distance between planes (111), $n_{\mathrm{c}}$ and $n_{\mathrm{m}}$ are the photonic crystal and surrounding medium refractive indexes respectively and $\theta_{\mathrm{ext}}$ is the external impinging angle with respect to the normal. In the case of the $\Gamma L$ direction in an opal (fcc lattice) the distance between planes is $d_{111}=\sqrt{2 / 3} a$ (or $d=2 a / \sqrt{3}$ ) where $a$ is the sphere diameter. To obtain this data, the equation above is written in terms of the internal angle and $n_{\mathrm{c}}$ is taken as the average refractive index of the spheres and the host dielectric while $n_{\mathrm{m}}$ is air. Thus

$$
\frac{a}{\lambda_{111}}=\frac{\sqrt{3}}{2 \cos \left(\theta_{\text {int }}\right) \sqrt{(n)}}
$$

with

$$
(n)=f n_{\text {silica }}+(1-f) n_{\text {host }},
$$

where $f$ is the filling fraction of the spheres (ca. 0.74 for touching spheres) and $\mathrm{n}_{\text {silica }}$ and $\mathrm{n}_{\text {host }}$ are the spheres (silica, 2.03) and host (air, 1.00) refractive index.

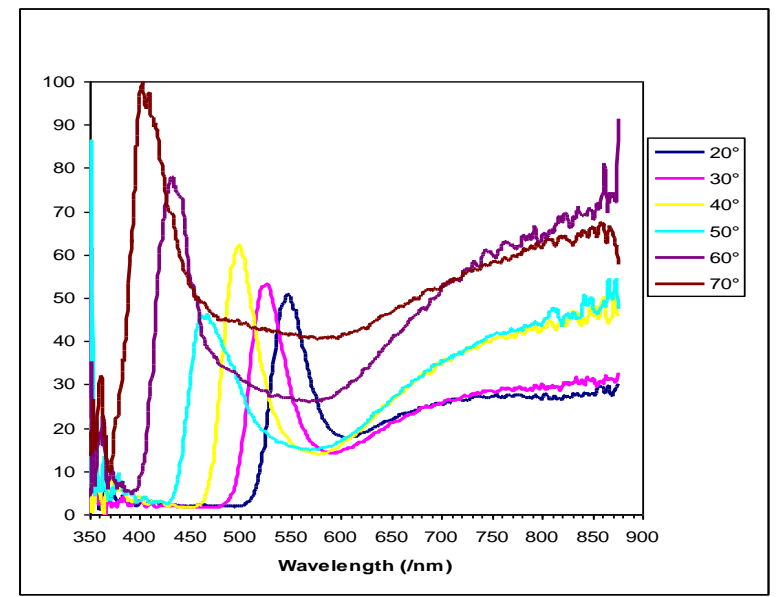

Fig. 4: Specular Reflectance spectra of the opal sample at different incidence angles of light

Bragg's law equation allows working with a very simple model to obtain some information about the photonic crystal. However this information is limited to the wavelength of the first pseudo gap and other characteristics such as the peak width remain unknown. Also, and due to symmetry features, some of the wave-vectors that lie on the Brillouin surface are not diffracted however. This fact is not reflected by Bragg's law and more complex approaches are needed to reproduce this behavior. Finally, the results obtained with Bragg's law will strongly depend on the dielectric constant we choose for the photonic crystal. Here the average dielectric constant was used but this choice is not always valid. We need use the effective refractive index. The RI is not a well defined parameter for a photonic crystal. Its value can be extracted from the slope of the bands and it may drastically differ for each frequency and propagation direction. However, the RI is relatively well defined at low energies and some calculations can be done assuming a constant value. The effective dielectric constant $\left(\varepsilon_{\text {eff }}\right)$ of a opal was taken as the 
average value of the materials forming the lattice. The volume fractions for each sample were deduced by means of optical measurements, specifically, studying the position of the first order diffraction peak in the $\Gamma \mathrm{L}$ direction. For this purpose Bragg's law should be expressed in terms of the volume fraction and the sphere diameter (known beforehand). The volume fraction for an fcc structure is

$$
f=\frac{2 \pi d^{3}}{3 a^{3}} \Rightarrow a=d\left(\frac{2 \pi}{3 f}\right)^{1 / 3}
$$

For our sample, the theoretical reflectance spectra, calculated with the experimentally determined particle size and refractive index values, give excellent agreement for the band gap positions. With a particle diameter of $253 \mathrm{~nm}$ and a refractive index of 1.37 for silica sphere, the theoretical band gap position and the experimental value is in good agreement (Fig. 5). When the angle of light incidence with respect to the (111) plane of the multilayer crystal, h, decreases, the first order diffraction peak shifts to lower wavelengths. The reflectance increases in the whole wavelength range, which is due to the increased background scattering (caused by the crystal defects or the substrate), as the light crosses increasing widths of the sample. From the positions of the band gap $\left(\lambda_{\max }\right)$ at the different angles (Fig. 5), the angular dispersion can be calculated. Figure 5 is a plot of the band gap positions $\left(\lambda_{\max }\right)$ as a function of $\theta$ angles for the sample. This plot is a representation of the band gap angular dispersion data in terms of the Bragg equation. The curve in Fig. 5 is calculated with the Bragg equation

$$
\lambda_{\max }=2 \sqrt{\frac{2}{3}} D \sqrt{\left(n_{e f f}^{2}-\sin ^{2 \theta}\right)},
$$

where $\mathrm{D}$ is the lattice parameter (or sphere diameter) $(\mathrm{D} \sim 0.816 \mathrm{~d}$ for an fcc crystal), $\mathrm{n}$ is the effective refractive index ( $n_{\text {eff. }} \sim 1.37$, assuming $74 \%$ volume filling of the silica balls), and $\theta$ is the angle between the incident light and the $\{111\}$ plane of the crystal.

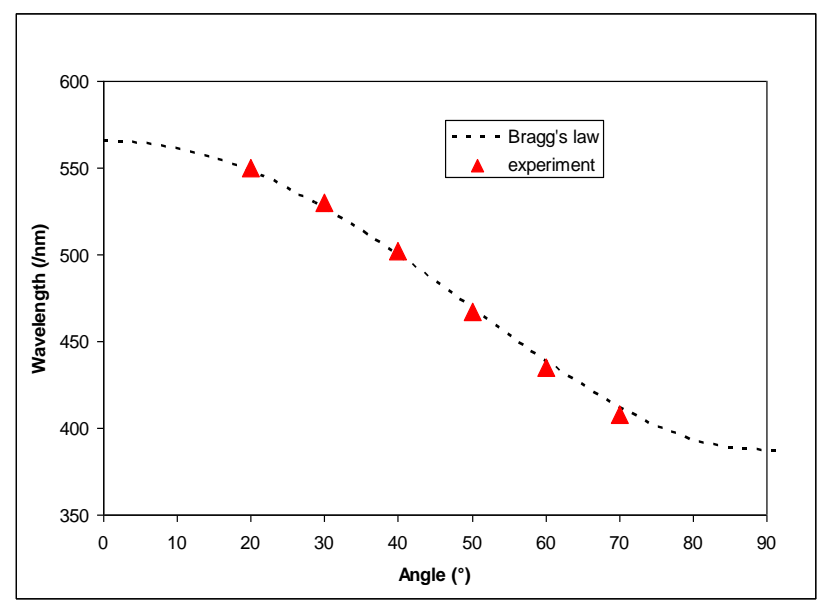

Fig. 5: Experimental band-gap of the Bragg peak of opal crystals obtained from $253 \mathrm{~nm}$ silica spheres, compared with the theoretical expectations of the band-gap in the $\Gamma L$ direction

\subsubsection{Self-activated luminescence of pure silica opals}

Under excitation of $400 \mathrm{~nm}$, in the luminescence spectra exicts, we observed a broad emission band from opal crystal samples (Fig. 6). This band is the self-activated luminescence of pure silica opal. It is original from R-OH group which is existence in the sample prepared from 
TEOS without heat treatment. Figure 7 shows the PL of opal crystal with CdSe/ZnS QDs in the different observed directions. An observed emission band at $585 \mathrm{~nm}$ is the emission of $\mathrm{CdSe} / \mathrm{ZnS}$ QDs. The emission of self-activated opal is decreased due to strength emission of QDs. It seems that the QDs are not yes enter into the opal structure.

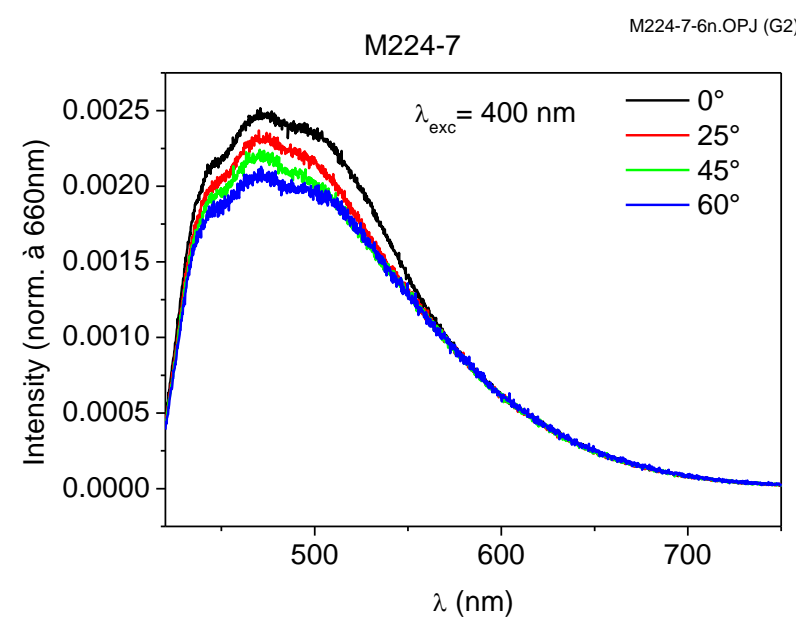

Fig. 6: PL spectra of opal photonic crystal sample under $400 \mathrm{~nm}$ excitation

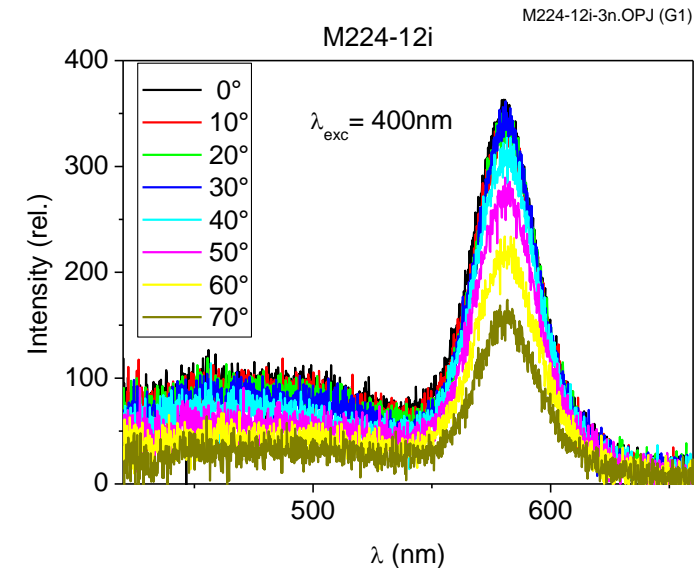

Fig.7: PL spectra of opal crystal with CdSe/ZnS QDs under $400 \mathrm{~nm}$ excitation

\section{CONCLUSION}

We prepared with of opal photonic crystals. Spectral position and width of the Bragg diffraction from the (111) planes in opals give information about lattice parameter, filling fraction, effective dielectric constant and crystal quality, as shown in agreement between theoretical calculation and experimental observation. Bragg's law is a good approximation for a first study of the pseudo gap behavior of an opal around the $\Gamma \mathrm{L}$ direction when refractive indices and their contrast are not high. The optical properties of the opals made of silica spheres are determined both by the Bragg diffraction inherent to the ordered structure. Photoluminescence spectra show that the QDs are not yes enter into opal photonic crystal. The method of infiltrating QDs into this photonic structure is necessary to be developed in our lab. 


\section{ACKNOWLEDGEMENT}

We would like to thank Profs. Nguyen Van Hieu and Phan Hong Khoi for multiple discussions. This work was financially supported by the Vietnamese Foundation for Basic Research (2006) and Bilateral Scientific Cooperation Project CNRS, Université Pierre et Marie Curie (France) and VAST (2006). The authors are grateful to the Key State Laboratory of Institute of Sciences Material of VAST. Thank for Dr. Nguyen Hong Quang and Do Hung Manh who provide the access to the FE- SEM instrument.

\section{REFERENCES}

1. Jeong-Ho Park, Won San Choi, Hye Young Koo, Jae-Chul Hong, and Dong-Yu Kim (2006), Langmuir, vol. 22, pp. 94-100.

2. Vlasov, Y.A., Bo, X.Z., Sturm, J.C., and Norris, D.J. (2001), Nature, vol. 414, pp. 289-293.

3. Park, S.H. and Xia, Y. (1999), Langmuir, vol. 15, p. 266.

4. Murray, C.B., Norris, D.J., and Bawendi, M.G. (1993), J. Am. Chem. Soc., vol. 115, p. 8706.

5. Murray, C.B., Kagan, C.R., and Bawendi, M.G. (2000), Annu. Rev. Mater. Sci., vol. 30, p. 545.

6. Eisler, H.-J., Sundar, V.C., Bawendi, M.G., Walsh, M., Smith, H.I., and Klimov, V. (2002), App. Phys. Lett., vol. 80, p. 4614.

7. Chan, Y., Caruge, J.M., Snee, P.T., and Bawendi, M.G. (2004), App. Phys. Lett., vol. 85, p. 2460.

8. Brokmann, X., Giacobino, E., Dahan, M., and Hermier, J.P. (2004), App. Phys. Lett., vol. 85, p. 712.

9. Parak, W.J., Gerion, D., Pellegrino, T., Zanchet, D., Micheel, C., Williams, S.C., Boudreau, R., Le Gros, M.A., Larabell, C.A., and Alivisatos, A.P. (2003), Nanotechnology, vol. 14, p. R15.

10. Blanco, A., et al. (2000), Nature, vol. 405, pp. 437-440.

11. John D. Joannopoulos (2001), Nature, vol. 414, pp. 257-258.

12. Stöber, W., Fink, A., and Bohn, E. J. (1968), Colloid Interface Sci., vol. 26, p. 62.

13. Jeong- Ho Park, Won San Choi, Hye Young Koo, and Dong-Yu Kim (2005), Advanced Materials, vol. 17(7), pp. 879-885. 